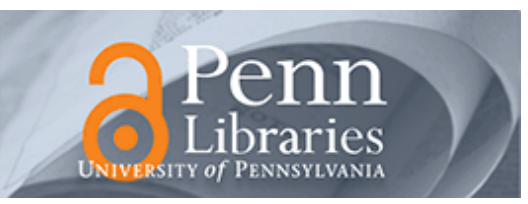

University of Pennsylvania

ScholarlyCommons

March 1997

\title{
Lateral stiffness: A new nanomechanical measurement for the determination of shear strengths with friction force microscopy
}

\author{
Robert W. Carpick \\ University of Pennsylvania, carpick@seas.upenn.edu \\ D. F. Ogletree \\ University of California \\ Miguel Salmeron \\ University of California
}

Follow this and additional works at: https://repository.upenn.edu/meam_papers

\section{Recommended Citation}

Carpick, Robert W.; Ogletree, D. F.; and Salmeron, Miguel, "Lateral stiffness: A new nanomechanical measurement for the determination of shear strengths with friction force microscopy" (1997).

Departmental Papers (MEAM). 95.

https://repository.upenn.edu/meam_papers/95

Copyright (1997) American Institute of Physics. This article may be downloaded for personal use only. Any other use requires prior permission of the author and the American Institute of Physics. Reprinted in Applied Physics Letters, Volume 70, Issue 12, March 1997, pages 1548-1550.

NOTE: At the time of publication, author Robert W. Carpick was affiliated with the University of Wisconsin. Currently (June 2007), he is a faculty member in the Department of Mechanical Engineering and Applied Mechanics at the University of Pennsylvania. Publisher URL: http://dx.doi.org/10.1063/1.118639

This paper is posted at ScholarlyCommons. https://repository.upenn.edu/meam_papers/95

For more information, please contact repository@pobox.upenn.edu. 


\title{
Lateral stiffness: A new nanomechanical measurement for the determination of shear strengths with friction force microscopy
}

\author{
Abstract \\ We present a technique to measure the lateral stiffness of the nanometer-sized contact formed between \\ a friction force microscope tip and a sample surface. Since the lateral stiffness of an elastic contact is \\ proportional to the contact radius, this measurement can be used to study the relationship between \\ friction, load, and contact area. As an example, we measure the lateral stiffness of the contact between a \\ silicon nitride tip and muscovite mica in a humid atmosphere (55\% relative humidity) as a function of \\ load. Comparison with friction measurements confirms that friction is proportional to contact area and \\ allows determination of the shear strength.
}

\section{Keywords}

microscopy, friction, shear, measuring methods, surfaces, elasticity, mica, humidity, silicon compounds, atomic force microscopy, sliding friction, shear strength, mechanical contact, mechanical variables measurement

\section{Comments}

Copyright (1997) American Institute of Physics. This article may be downloaded for personal use only. Any other use requires prior permission of the author and the American Institute of Physics. Reprinted in Applied Physics Letters, Volume 70, Issue 12, March 1997, pages 1548-1550.

NOTE: At the time of publication, author Robert W. Carpick was affiliated with the University of Wisconsin. Currently (June 2007), he is a faculty member in the Department of Mechanical Engineering and Applied Mechanics at the University of Pennsylvania. Publisher URL: http://dx.doi.org/10.1063/1.118639 


\title{
Lateral stiffness: A new nanomechanical measurement for the determination of shear strengths with friction force microscopy
}

\author{
R. W. Carpick, ${ }^{\text {a) }}$ D. F. Ogletree, and M. Salmeron ${ }^{\text {b) }}$ \\ Materials Sciences Division, Lawrence Berkeley National Laboratory, Berkeley, California 94720
}

(Received 21 November 1996; accepted for publication 20 January 1997)

\begin{abstract}
We present a technique to measure the lateral stiffness of the nanometer-sized contact formed between a friction force microscope tip and a sample surface. Since the lateral stiffness of an elastic contact is proportional to the contact radius, this measurement can be used to study the relationship between friction, load, and contact area. As an example, we measure the lateral stiffness of the contact between a silicon nitride tip and muscovite mica in a humid atmosphere (55\% relative humidity) as a function of load. Comparison with friction measurements confirms that friction is proportional to contact area and allows determination of the shear strength. (C) 1997 American Institute of Physics. [S0003-6951(97)01412-5]
\end{abstract}

The friction force microscope (FFM $)^{1}$ has emerged as an important tool to study nanotribology - the atomic scale origins of friction, adhesion, lubrication, and wear. ${ }^{2}$ Recent observations indicate that at low loads, the FFM tip can form a single asperity contact with a surface ${ }^{3-6}$ and wearless interfacial sliding occurs. Friction appears to scale with load in proportion to the area of contact as predicted for a continuous, elastic, single asperity contact. In other words,

$$
F_{f}=\tau A=\tau \pi a^{2},
$$

where $F_{f}$ is the frictional force, $A$ the contact area, $a$ the contact radius, and $\tau$ the shear strength (shear force/area), in contrast to the macroscopic observation of friction being proportional to load (due to multiple asperity contact ${ }^{7}$ and/or wear or plastic deformation ${ }^{8}$ ). However, contact area is not directly measured with FFM, so a contact mechanical model must be chosen to properly investigate the relationship between friction and contact area. The particular model utilized, such as the Hertz ${ }^{9}$ or Johnson-Kendall-Roberts ${ }^{10}$ model, depends upon the strength and range of the tipsample interaction forces ${ }^{11}$ (among other things), which is uncertain in each case. The contact area-load relation for a single asperity also depends upon the tip shape, as demonstrated experimentally by Carpick et al. ${ }^{6}$ Furthermore, if the shear strength is not independent of load (pressure), then the load dependence of shear strength and contact area become convoluted. ${ }^{4}$ As well, the models used neglect the effect of lateral forces upon the contact area, yet this may indeed be a significant effect ${ }^{12,13}$ to explore. For these reasons, an independent measurement related to the contact area is desirable.

Contact stiffness is defined as the amount of force per unit displacement required to compress an elastic contact in a particular direction, has the units of $\mathrm{N} / \mathrm{m}$, and is essentially the "spring constant" of the contact. For example, the normal stiffness is given by $\kappa=d L / d z$, where $L$ is the applied load (normal force), and $z$ is the elastic penetration depth. In the Hertz case ${ }^{9}$ (an elastic sphere-plane contact),

$$
\kappa_{\text {contact }}=2 a E^{*},
$$

\footnotetext{
a) Also at Department of Physics, University of California at Berkeley, Berkeley, California.

${ }^{b)}$ Author to whom correspondence should be sent. Phone: (510) 486-6704; fax (510) 486-4995. Electronic mail: salmeron@stm.lbl.gov
}

where $E^{*}=\left[\left(1-\nu_{1}^{2}\right) / E_{1}+\left(1-\nu_{2}\right)^{2} / E_{2}\right]^{-1} ; E_{1}$ and $E_{2}$ are the Young's moduli of the sphere and plane, respectively, and $\nu_{1}$ and $\nu_{2}$ the respective Poisson's ratios. From Eq. (2), the normal stiffness is directly proportional to the contact radius, which for the Hertz case is given by $a=\left(3 R L / 4 E^{*}\right)^{1 / 3}$, where $R$ is the sphere radius (the sphere is approximated as a paraboloid). Typically the contact strain is concentrated within a volume of the order $a^{3}$.

With FFM, the plane corresponds to the sample, and the sphere corresponds to the tip. In addition, the sphere is attached to a spring, i.e., the cantilever, which has its own stiffness (the normal spring constant $\kappa_{\text {lever }}$ ). The cantilever and the contact are thus two springs in series [Fig. 1(a)]. For nanometer-sized contacts between common materials like metals and ceramics, stiffness values are roughly 50-500 $\mathrm{N} / \mathrm{m}$. However, the normal stiffness of typical commercial FFM cantilevers, $\kappa_{\text {lever }}$, is on the order of $0.01-1 \mathrm{~N} / \mathrm{m}$. Thus nearly all the elastic compression is taken up by the lever and not the contact, so the measurement is relatively insensitive to $\kappa_{\text {contact }}$. Notably, Pethica and co-workers ${ }^{14}$ have designed a substantially modified scanning force microscope using custom-made cantilevers where a magnetic force is directly applied to the tip. With this setup, the normal stiffness can be sensitively measured.

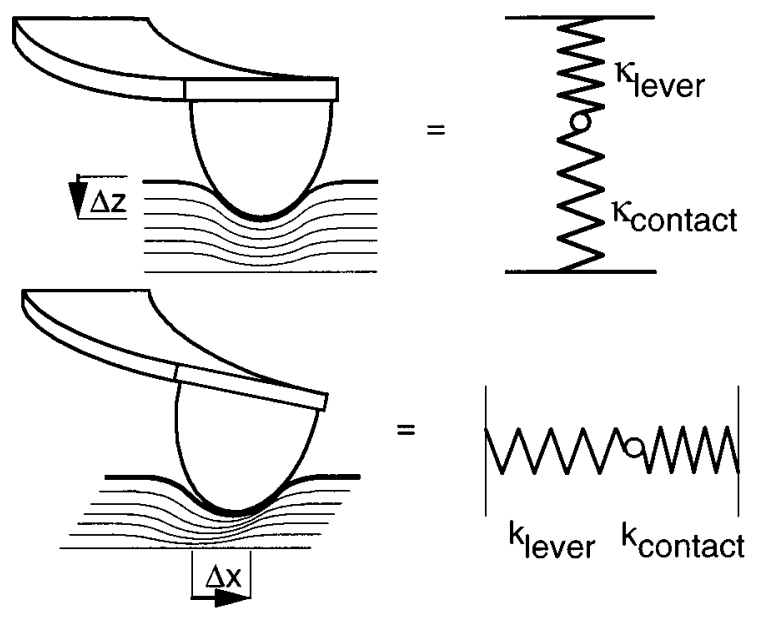

FIG. 1. Model showing normal and lateral stiffnesses in FFM. 


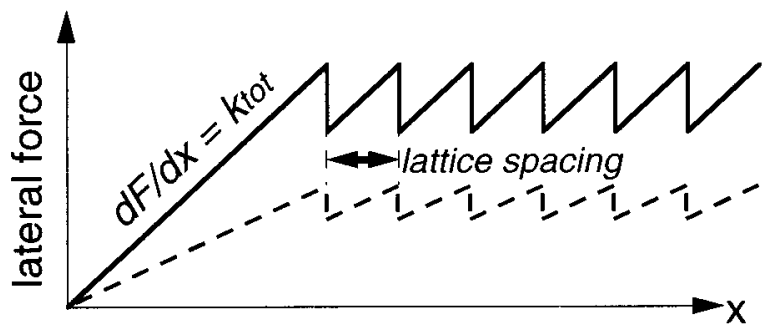

FIG. 2. Lateral force signal vs lateral displacement $(x)$. Solid line: a relatively stiff contact. Dashed line: a softer contact-there is less cantilever bending per unit displacement since the contact is being substantially compressed.

However, the typical lateral stiffness of commercial FFM cantilevers, $k_{\text {lever }}$, is around $50-200 \mathrm{~N} / \mathrm{m},{ }^{15}$ i.e., of the same order as the lateral contact stiffness, $k_{\text {contact }}$, so typical cantilevers can accurately measure variations in the lateral stiffness of nanometer-sized contacts, i.e.,

$$
\frac{d F_{\text {lateral }}}{d x}=k_{\text {tot }}=\left[\frac{1}{k_{\text {lever }}}+\frac{1}{k_{\text {contact }}}\right]^{-1},
$$

where $F_{\text {lateral }}$ is the lateral force (cantilever torsion), and $x$ is the lateral displacement [Fig. 1(b)]. For a sphere-plane contact, $k_{\text {contact }}$ is given by ${ }^{19}$

$$
k_{\text {contact }}=8 G^{*} a,
$$

where $G^{*}=\left[\left(2-\nu_{1}\right) / G_{1}+\left(2-\nu_{2}\right) / G_{2}\right]^{-1}$. Here $G_{1}$ and $G_{2}$ are the tip and sample shear moduli, respectively. Again, $k_{\text {contact }}$ is directly proportional to the contact radius. A further advantage is that Eq. (4) holds, regardless of the tip-sample interaction forces, ${ }^{16}$ unlike the analogous equation for normal stiffness, Eq. (2), which must be modified for nonHertzian contacts.

A simple explanation of Eq. (4) is obtained by considering an applied lateral force $d F_{\text {lateral }}$ at fixed load, i.e., applying a lateral stress $d \sigma$ over the contact area $A$, producing a proportional strain $d \epsilon$, where $d \epsilon \propto d x / a$ (since $a$ is the length scale of the stress distribution). Stress and strain are related by Hooke's Law, $d \sigma=G \times d \varepsilon$, where $G$ is the shear modulus, appropriate for the direction of the applied stress considered here. Hence $d F_{\text {lateral }} / A \propto G \times d x / a$, giving $d F_{\text {lateral }} / d x$ $\propto G \times a$ as in Eq. (4). Note the simplifying assumption that the contact radius is not affected by the lateral displacement $d x$. This is reasonable and expected for small lateral displacements. ${ }^{16}$ In the case of normal stiffness, the normal displacement $d z$ does change the contact radius, which essentially explains why normal stiffness is not generally proportional to contact radius (except in the Hertz case). The relation between lateral stiffness and energy dissipation from friction has been discussed by Colchero et al. ${ }^{17}$

As long as there is finite static friction between the tip and sample, the lateral stiffness can be measured. Consider the lateral force response of a cantilever as it scanned across a sample (Fig. 2). Typically, atomic scale stick-slip behavior is preceded by an initial sticking portion, the slope of which corresponds to $d F_{\text {lateral }} / d x=k_{\text {tot }}$, the total lateral stiffness. To measure this slope accurately, the relative lateral position between the cantilever base and the sample is sinusoidally modulated with an amplitude small enough, typically a few

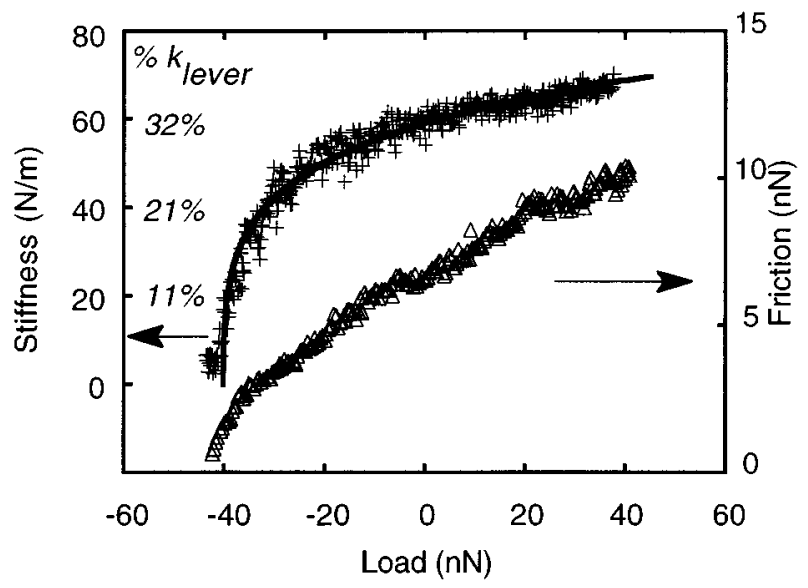

FIG. 3. Crosses: lateral stiffness ( $\left.k_{\text {tot }}\right)$ vs load data. Solid line: a fit of the shifted Hertz model [Eq. (5)]. As load increases, $k_{\text {tot }}$ asymptotically approaches $k_{\text {lever }},(\sim 190 \mathrm{~N} / \mathrm{m}$, from the fit $)$, although, even at the maximum load, $k_{\text {tot }} \sim 35 \% k_{\text {lever }}$. Triangles: $F_{\mathrm{f}}$ vs load, acquired shortly after the stiffness measurement.

angstroms, to avoid slip even at low loads. A lock-in amplifier is used to measure the amplitude of the lateral force response over a range of loads. If slip occurs, a significant out-of-phase lock-in response results. A two channel lock-in can monitor the out-of-phase component, to discard measurement points where slip occurred. This will be discussed in more detail elsewhere ${ }^{18}$ and was not necessary for this example. The in-phase amplitude $\left(d F_{\text {lateral }}\right)$ divided by the amplitude of relative displacement $(d x$, determined by accurately knowing the piezo response calibration) corresponds to the total lateral stiffness of the system, $k_{\text {tot }}$ [Eq. (3)]. Using a similar setup, Colchero et al. ${ }^{19}$ measured friction by using a large lateral displacement amplitude so that sliding took place. For our measurements, a silicon nitride Digital Instruments ${ }^{20}$ cantilever with a nominal normal force constant $\sim 0.58 \mathrm{~N} / \mathrm{m}$ was used.

Lateral stiffness $\left(k_{\text {tot }}\right)$ was measured versus load for the tip contacting freshly cleaved muscovite mica in humid atmosphere $[\sim 55 \%$ relative humidity (RH) $]$ (Fig. 3 - crosses). The $k_{\text {tot }}$ shows a distinct load dependence with a good signal to noise ratio. The fit (Fig. 3 - solid line) indicates how $k_{\text {tot }}$ should vary with load (also fitting a value for $k_{\text {lever }}$ ), using the Hertz theory with the load axis shifted by the critical load $L_{\mathrm{c}}$ (pull-off force),

$$
a=\left[\frac{3 R}{4 E^{*}}(L+L c)\right]^{1 / 3} .
$$

This dependence is predicted by Fogden and White ${ }^{21}$ for an elastic contact in the presence of capillary condensation for appropriate values of the elastic constants, tip radius, and relative humidity. Using their model, we have determined that Eq. (5) should apply in our case. A more detailed discussion of this approach has been presented elsewhere. ${ }^{4}$ Note from Fig. 3 that $k_{\text {tot }}$ only reaches $35 \%$ of $k_{\text {lever }}$ at the highest load; the contact deformation is equal to or greater than the lateral lever deformation at loads and conditions typically encountered in FFM experiments. ${ }^{17}$

We emphasize that no model needs to be chosen to determine the shear strength $\tau$ of the contact. To do this, fric- 


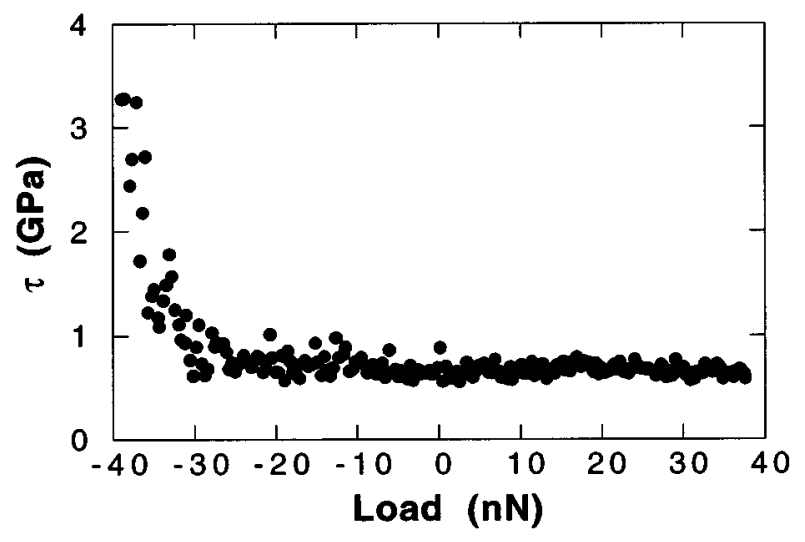

FIG. 4. The total stiffness $\tau$ vs load from the stiffness and friction data in Fig. 3.

tion was measured as a function of load immediately after the stiffness measurement (Fig. 3 - triangles) using a standard technique described elsewhere. ${ }^{3,6,22}$ To see how $\tau$ varies with load, Eqs. (1), (3), and (4) are combined to give

$$
\tau=\frac{64 G^{* 2} F_{f}}{\pi k_{\text {contact }}^{2}} .
$$

We calculate and plot $64 \cdot G^{* 2} \cdot F_{f} / \pi \cdot k_{\text {contact }}^{2}$ at each load value from the separately acquired measurements of friction and stiffness (Fig. 4). The shear strength $\tau$ appears to be independent of load with a value of $\sim 680 \mathrm{MPa}$, except near pull-off $(\sim-40 \mathrm{nN})$. We believe this low-load anomaly is due to water or hydrocarbon contaminants between the tip and sample which lead to low stiffness values at low loads, ${ }^{23}$ and/or slippage occurring at low loads. We emphasize that this value for $\tau$ is an estimate as we rely upon bulk values of the elastic constants. These values are somewhat uncertain for the tip material, silicon nitride, because it is produced by chemical vapor deposition resulting in an amorphous structure with uncertain stoichiometry and residual stress. Our most informed estimate is $G_{1}=61 \mathrm{GPa}, \nu_{1}=0.27 .^{24,25}$ The bulk values for mica are $G_{2}=13.5 \mathrm{GPa}, \nu_{1}=0.10 .^{26} \mathrm{Mica}$, having a significantly smaller shear modulus, influences $G^{*}$ more strongly, giving $G^{*}=5.9 \mathrm{GPa}$. It is not known if the bulk values for elastic constants are valid at the nanometer scale (which could in fact be tested with this method), but recent nanomechanical measurements of gold indicate approximate agreement with bulk values. ${ }^{27,28}$ Unlike modeldependent methods, the tip radius is not needed to calculate $\tau$. Note that if the tip is not parabolic, the coefficient of $G^{*} a$ in Eq. (4) would be different. We confirmed this tip to be parabolic using the sharp edges of a faceted $\mathrm{SrTiO}_{3}(305)$ sample as described previously. ${ }^{6,29}$ A certain value for $k_{\text {lever }}$ was assumed above. Methods to determine $k_{\text {lever }}$ if calibration uncertainties exist will be discussed elsewhere, along with detailed comments on error analysis and further measurements at other humidities and in ultrahigh vacuum (UHV). ${ }^{18}$
In conclusion, we have described a fast and straightforward technique to determine the shear strength of a FFM tip-sample contact independent of contact mechanics models, by measuring the lateral contact stiffness. In general, friction and lateral stiffness measurements are complementary techniques which should be employed in tandem when studying nanotribology with FFM.

The authors gratefully acknowledge Professor K. L. Johnson for useful discussions and encouragement. R. W. C. acknowledges support from the Natural Sciences and Engineering Research Council of Canada. This work was supported by the Director, Office of Energy Research, Basic Energy Sciences, Materials Division of the U.S. Department of Energy under Contract No. DE-AC03-76SF00098.

${ }^{1}$ C. M. Mate, G. M. McClelland, R. Erlandsson, and S. Chiang, Phys. Rev. Lett. 59, 1942 (1987).

${ }^{2}$ Fundamentals of Friction: Macroscopic and Microscopic Processes, edited by I. L. Singer and H. M. Pollock (Kluwer, Dordrecht, 1992), Vol. 220.

${ }^{3}$ J. Hu, X.-D. Xiao, D. F. Ogletree, and M. Salmeron, Surf. Sci. 327, 358 (1995).

${ }^{4}$ U. D. Schwarz, W. Allers, G. Gensterblum, and R. Wiesendanger, Phys. Rev. B 52, 14976 (1995).

${ }^{5}$ E. Meyer, R. Luthi, L. Howald, M. Bammerlin, M. Guggisberg, and H.-J. Guntherodt, J. Vac. Sci. Technol. B 14, 1285 (1996).

${ }^{6}$ R. W. Carpick, N. Agraï, D. F. Ogletree, and M. Salmeron, J. Vac. Sci. Technol. B 14, 1289 (1996).

${ }^{7}$ J. A. Greenwood, in Fundamentals of Friction, edited by I. L. Singer and H. M. Pollock (Kluwer, Dordrecht, 1992), p. 111.

${ }^{8}$ F. P. Bowden and D. Tabor, Friction and Lubrication of Solids: Part I (Oxford University Press, 1950).

${ }^{9}$ K. L. Johnson, Contact Mechanics (University Press, Cambridge, 1987).

${ }^{10}$ K. L. Johnson, K. Kendall, and A. D. Roberts, Proc. R. Soc. London, Ser. A 324, 301 (1971).

${ }^{11}$ K. L. Johnson, Langmuir 12, 4510 (1996).

${ }^{12}$ A. R. Savkoor, in Ref. 7, p. 111.

${ }^{13}$ K. L. Johnson, Proc. R. Soc. London Ser. A 543, 163 (1997).

${ }^{14}$ S. P. Jarvis, A. Oral, T. P. Weihs, and J. B. Pethica, Rev. Sci. Instrum. 64, 3515 (1993).

${ }^{15}$ D. F. Ogletree, R. W. Carpick, and M. Salmeron, Rev. Sci. Instrum. 67, 3298 (1996)

${ }^{16}$ K. L. Johnson (personal communication).

${ }^{17}$ J. Colchero, A. M. Baro, and O. Marti, Trib. Lett. 2, 327 (1996).

${ }^{18}$ R. W. Carpick, D. F. Ogletree, and M. Salmeron (unpublished).

${ }^{19}$ J. Colchero, M. Luna, and A. M. Baro, Appl. Phys. Lett. 68, 2896 (1996).

${ }^{20}$ Nanoprobe, Digital Instruments, Santa Barbara, CA.

${ }^{21}$ A. Fogden and L. R. White, J. Colloid Interface Sci. 138, 414 (1990).

${ }^{22}$ R. W. Carpick, N. Agraï, D. F. Ogletree, and M. Salmeron, Langmuir 12, 3334 (1996).

${ }^{23}$ M. Binggeli and C. M. Mate, Appl. Phys. Lett. 65, 415 (1994).

${ }^{24}$ J. A. Taylor, J. Vac. Sci. Technol. A 9, 2464 (1991).

${ }^{25}$ M. Tortonese, Park Scientific Instruments Inc., Sunnyvale, CA (personal communication).

${ }^{26}$ L. E. McNeil and M. Grimsditch, J. Phys. Condens. Matter. 5, 1681 (1992).

${ }^{27}$ N. Agrait, G. Rubio, and S. Vieira, Phys. Rev. Lett. 74, 3995 (1995).

${ }^{28}$ P. Tangyunyong, R. C. Thomas, J. E. Houston, T. A. Michalske, R. M. Crooks, and A. J. Howard, Phys. Rev. Lett. 71, 3319 (1993).

${ }^{29}$ S. S. Sheiko, M. Möller, E. M. C. M. Reuvekamp, and H. W. Zandbergen, Phys. Rev. B 48, 5675 (1993). 